\title{
Highly Directional Small-Size Antenna Designed with Homogeneous Transformation Optics
}

\author{
Zuojia Wang, ${ }^{1,2}$ Lian Shen, ${ }^{1,2}$ Jun Chen, ${ }^{3}$ Huaping Wang, ${ }^{3}$ \\ Faxin $Y u^{3}$ and Hongsheng Chen ${ }^{1,2}$ \\ ${ }^{1}$ The Electromagnetics Academy at Zhejiang University, Zhejiang University, Hangzhou 310027, China \\ ${ }^{2}$ Department of Information Science and Electronic Engineering, Zhejiang University, Hangzhou 310027, China \\ ${ }^{3}$ School of Aeronautics and Astronautics, Zhejiang University, Hangzhou 310027, China \\ Correspondence should be addressed to Jun Chen; chnjun@zju.edu.cn, Huaping Wang; wang_hp2003@yahoo.com \\ and Hongsheng Chen; hansomchen@zju.edu.cn
}

Received 7 March 2014; Revised 23 April 2014; Accepted 27 April 2014; Published 21 May 2014

Academic Editor: Wenxing Li

Copyright (C) 2014 Zuojia Wang et al. This is an open access article distributed under the Creative Commons Attribution License, which permits unrestricted use, distribution, and reproduction in any medium, provided the original work is properly cited.

Achieving high directivity antenna usually requires a large size antenna aperture in traditional antenna design. Previous work shows that, with the help of metamaterials and transformation optics, a small size antenna can perform as high directivity as a large size antenna, but the material parameters are inhomogeneous and difficult to realize. In this paper, we propose a linear homogeneous coordinate transformation to design the small size antenna. Distinguishing from inhomogeneous transformation, we construct a regular polygon in virtual space and then divide it into several triangle segments. By applying linear homogeneous coordinate transformation, the antenna devices can be greatly compressed without disturbing the radiation patterns by using homogeneous metamaterial substrates. The material parameters of the antenna designed from this method are homogeneous and easy to fabricate. Square and hexagonal antenna structures are numerically demonstrated to illustrate the validity of our methodology.

\section{Introduction}

Nowadays the demand for compact radiators with sufficiently high directivity is rapidly increasing in many application areas. In particular, modern wireless telecommunication systems require compact antennas with high directivity, which is especially crucial when the radiating components have to be combined in large antenna arrays for satellites, airplanes, space vehicles, and so forth. However, it is a fundamental trade-off between the directivity factor and the antenna profile in traditional antenna design. Metamaterials, with their unusual electromagnetic properties, provide a feasible solution to reduce the size of the antenna but still keep the high directivity. Firstly known as left-handed materials (LHM), metamaterials have attracted great attention and offered great opportunity to the manipulation of electromagnetic (EM) fields. For example, metamaterials have been widely used for the designs of optical superlens $[1,2]$, invisibility cloak [3-5], illusion device [6, 7], super absorber [8], antenna
[9-12], and so forth. With inhomogeneous transformation optics whose transformation ratio relates to the position in each region, it is shown that the antenna size can be greatly reduced [13]. However, the material parameters are inhomogeneous and anisotropic, which are very difficult to realize.

In this paper, we present a novel design for smallsize directional antenna using a linear homogeneous transformation method whose transformation ratio is independent of the position. Distinguishing from inhomogeneous transformation, we construct a regular polygon in original space and then divide it into several triangle segments. By applying a linear homogeneous coordinate transformation, the antenna devices can be greatly compressed without disturbing the radiation patterns by using homogeneous metamaterial substrates. The simulated results of square and hexagonal antenna structures demonstrate the validity of our methodology, showing its potential applications in microwave antenna. 


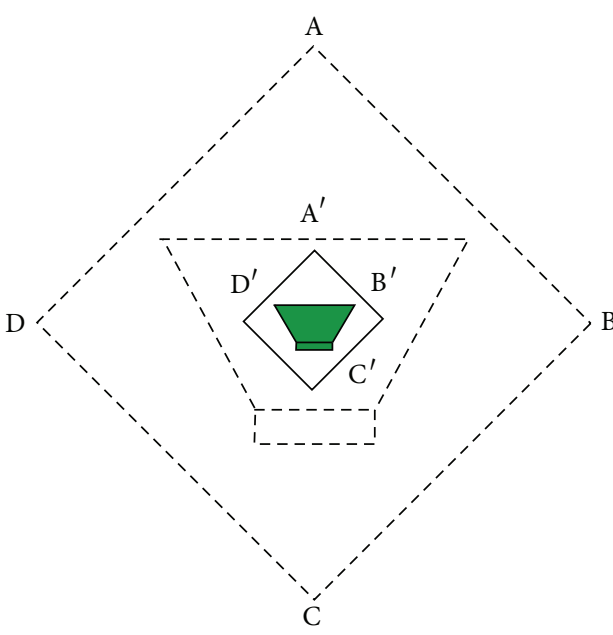

(a)

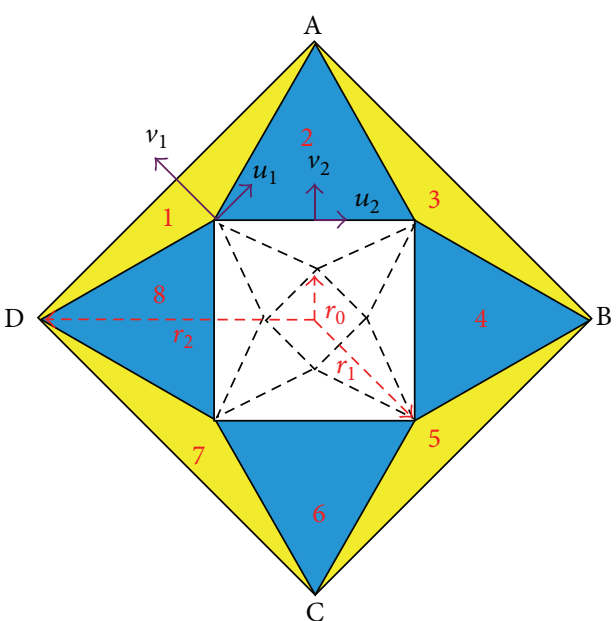

Segment I

Segment II

(b)

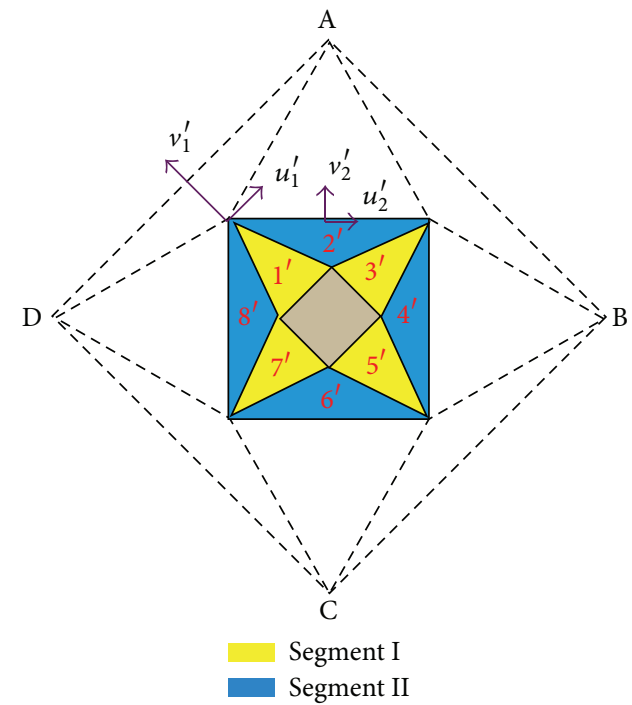

(c)

Figure 1: (a) The schematic of the square antenna system. A big square region (ABCD) with radius $r_{2}$ has been compressed to a small one $\left(\mathrm{A}^{\prime} \mathrm{B}^{\prime} \mathrm{C}^{\prime} \mathrm{D}^{\prime}\right)$ with radius $r_{0}$. Green region represents the antenna device compressed from a large size one described by dashed lines. (b) The square in the virtual coordinate space filled with isotropic material is divided into several triangle segments grouped by Segment I and Segment II. (c) The square metamaterial shell in the physical coordinate space is constructed by the transformed segments with homogeneous constitutive tensors.

\section{Results and Discussion}

For simplicity, we limit ourselves to two-dimensional case and TE incidence. As depicted in Figure 1, a big square free space (ABCD) with radius $r_{2}$ has been compressed to a small antenna core $\left(\mathrm{A}^{\prime} \mathrm{B}^{\prime} \mathrm{C}^{\prime} \mathrm{D}^{\prime}\right)$ with radius $r_{0}$. This can be achieved by a linear homogenous transformation [14]. Specifically, we construct another square with radius $r_{1}$ rotated at an angle of $\pi / 4$ compared to the outside one, as shown in Figure 1(b). The space between the two squares is then divided into many triangle segments. Due to the symmetric pattern shown in Figure 1(b), we group the triangle segments into two types, marked as Segment I (blue regions) and Segment II (yellow regions) with their local coordinate axes $\left(u_{1}, v_{1}, w_{1}\right)$ and $\left(u_{2}\right.$, $\left.v_{2}, w_{2}\right)$, respectively. The following linear transformation is applied in each segment along its local coordinate axes:

$$
\begin{aligned}
& u_{1}^{\prime}=\kappa_{u} u_{1}, \quad v_{1}^{\prime}=\kappa_{v} v_{1}, \quad w_{1}^{\prime}=w_{1}, \quad \text { for Segment I, } \\
& u_{2}^{\prime}=u_{2}, \quad v_{2}^{\prime}=\kappa v_{2}, \quad w_{2}^{\prime}=w_{2}, \quad \text { for Segment II, }
\end{aligned}
$$

where $\kappa=\left(r_{1} \cos (\pi / m)-r_{0}\right) /\left(r_{1} \cos (\pi / m)-r_{2}\right), \kappa_{v}=$ $\left(r_{1}-r_{0} \cos (\pi / m)\right) /\left(r_{1}-r_{2} \cos (\pi / m)\right), m=4$ for the square case, and $\kappa_{u}=r_{0} / r_{2}$ are the compression ratios of the space applicable for arbitrary regular polygonal. Through this 


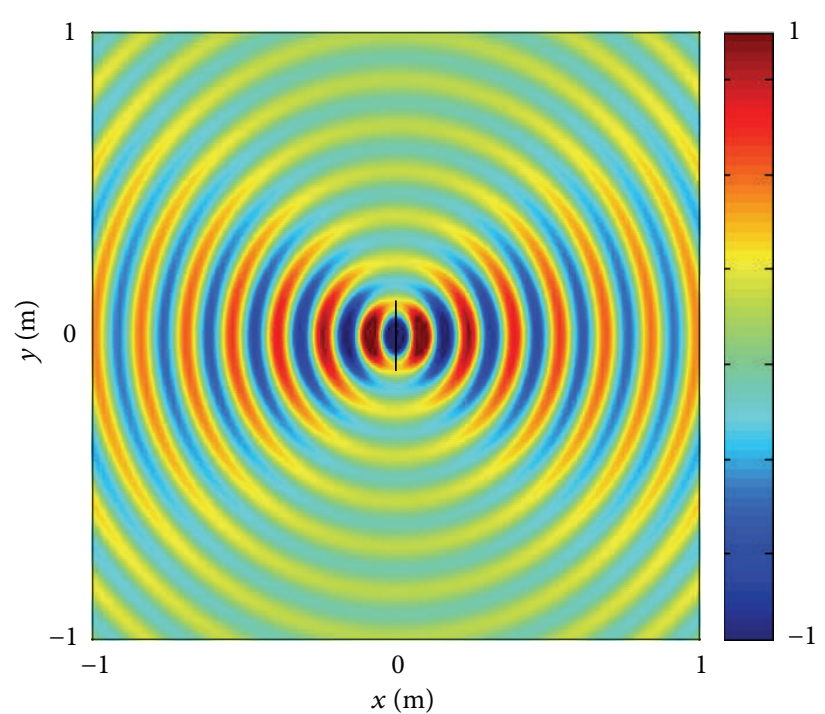

(a)

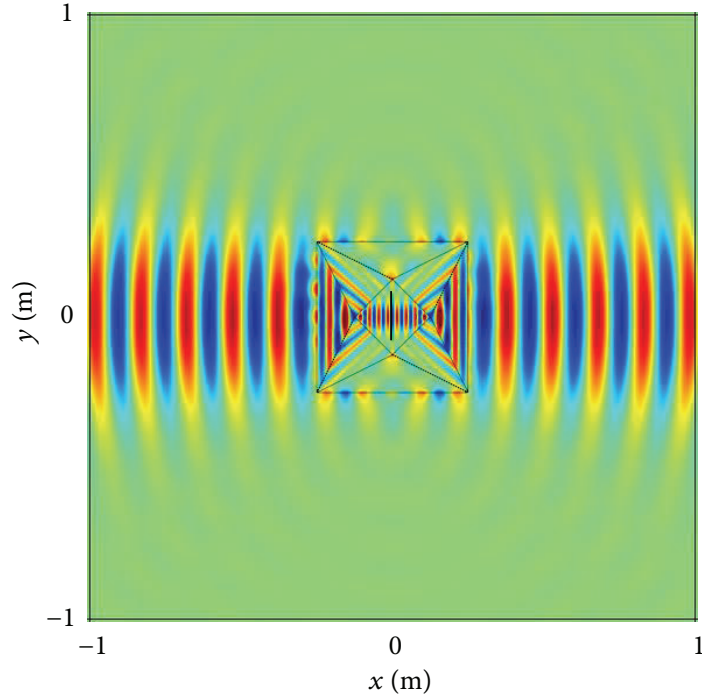

(b)

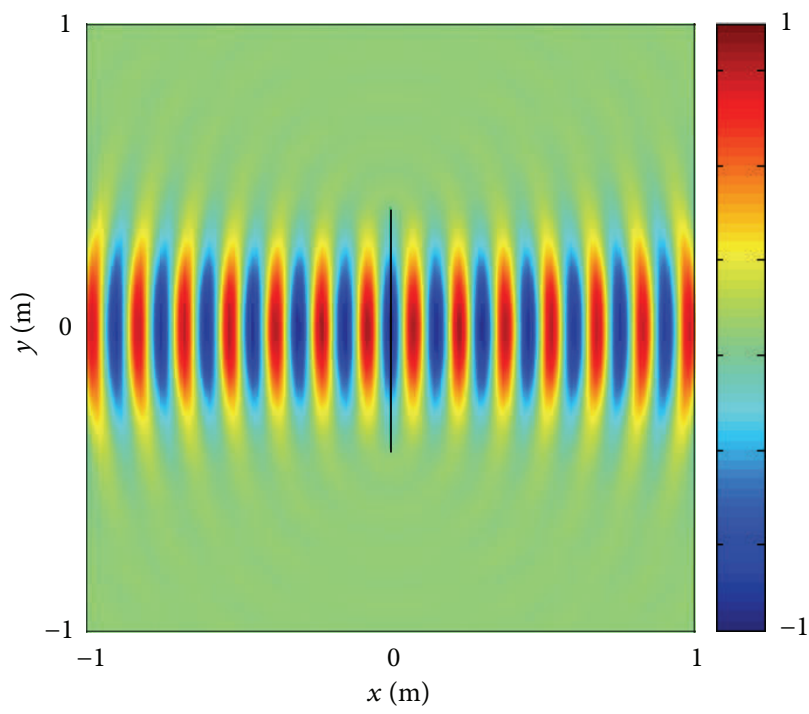

(c)

FIGURE 2: The $z$-directed electrical field distribution when a naked small current plane with width $w=0.16 \mathrm{~m}$ is located in (a) free space and (b) square metamaterial substrates, respectively. (c) $E_{z}$ field distribution while a large current plane with the width $0.8 \mathrm{~m}$ is radiating in free space.

transformation, the blue and yellow regions in Figure 1(b) are reversed to the space with the same colors shown in Figure 1(c), respectively. Meanwhile, the large square region (ABCD) is compressed to a small brown core. Hence properly designed compact antennas located in the brown core can effectively mimic large size ones and exhibit identical radiation patterns. In case of TE incident wave, the material parameters of the metamaterial shell can be obtained as

$$
\mu_{u}^{I^{\prime}}=\frac{\kappa_{u}}{\kappa_{v}}, \quad \mu_{v}^{I^{\prime}}=\frac{\kappa_{v}}{\kappa_{u}}, \quad \varepsilon_{w}^{I^{\prime}}=\frac{1}{\left(\kappa_{u} \kappa_{v}\right)},
$$

for Segment I,

$$
\begin{aligned}
& \mu_{u}^{I I^{\prime}}=\frac{1}{\kappa}, \quad \mu_{v}^{I I^{\prime}}=\kappa, \quad \varepsilon_{w}^{I I^{\prime}}=\frac{1}{\kappa}, \\
& \text { for Segment II, } \\
& \mu_{u}^{\text {core' }}=1, \quad \mu_{v}^{\text {core' }}=1, \quad \varepsilon_{w}^{\text {core' }}=\left(\frac{1}{\kappa_{u}}\right)^{2},
\end{aligned}
$$

for the antenna core.

The physical square region is therefore composed of several triangular segments with homogenous and anisotropic materials. It is interesting to see that the media making up the antenna substrates are homogeneous. Different from the 


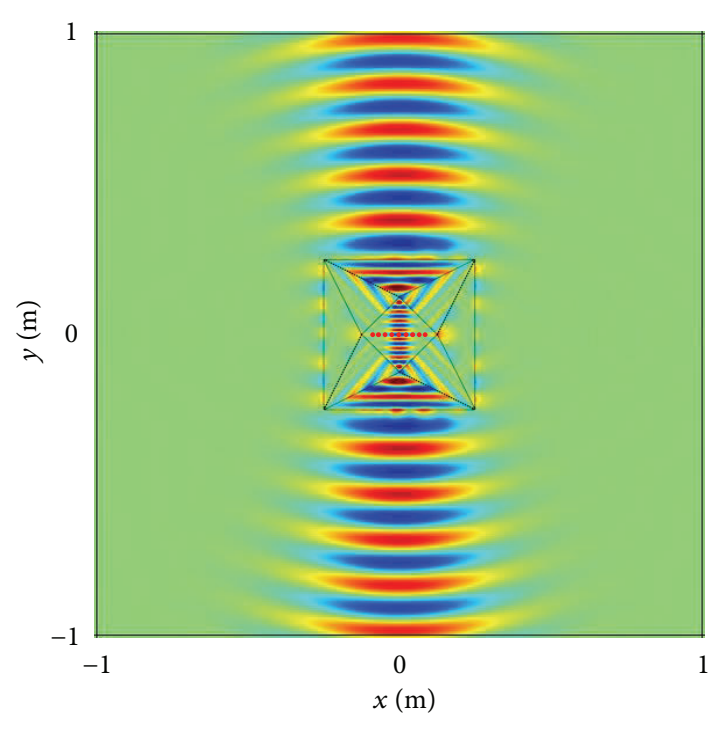

(a)

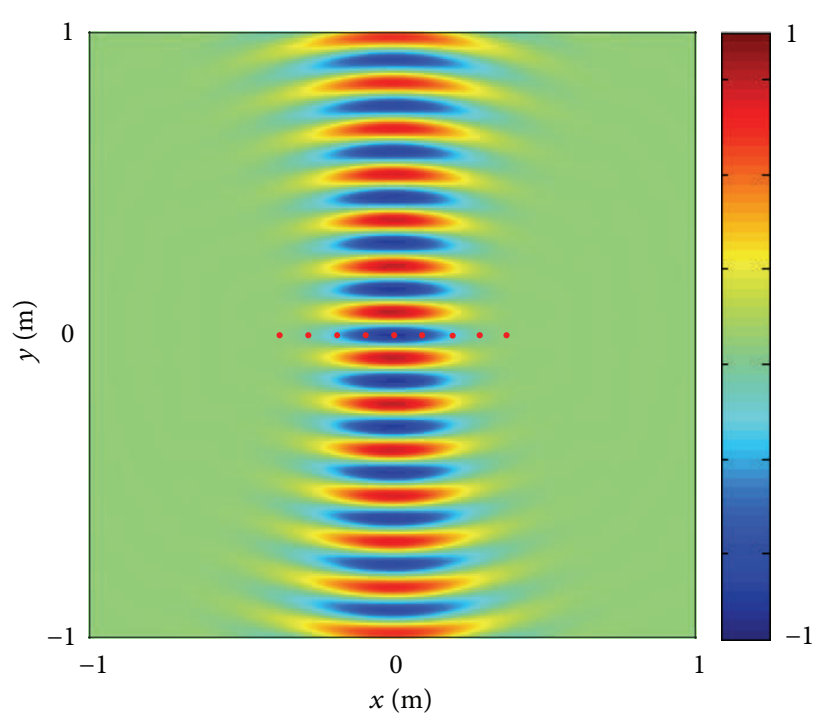

(b)

FIGURE 3: (a) $E_{z}$ field distribution of a small antenna array with nine elements embedded in square metamaterial shell. (b) $E_{z}$ field distribution of a large antenna array in free space.

inhomogeneous optical transformation scheme, the linear transformation applied here is very simple, yielding homogeneous constitutive parameters of metamaterial substrates. The virtual antenna is therefore mapped to a smaller one (colored by green in Figure 1(a)) with the compression ratio $r_{2} / r_{0}$. In addition, we set $r_{0}$ to be small enough so that the size of the antenna device can be significantly reduced. In the following part, we will give examples to illustrate how this approach reduces the device size without disturbing the radiation behavior. All the results are obtained from a commercial finite element modeling software (COMSOL) and the working frequency is set at $2 \mathrm{GHz}$.

Figure 2(a) depicts the simulated field distribution of a naked antenna excited by a surface current plane (with the width $16 \mathrm{~cm}$ ). In this case we set $r_{0}=0.125 \mathrm{~m}, r_{1}=2 \sqrt{2} r_{0}$, and $r_{2}=5 r_{0}$. In Figure 2(b), we plot the electrical field distribution of the same current plane (with the width $0.16 \mathrm{~m}$ ) embedded in a metamaterial shell. The coated layer consists of homogeneous metamaterials with $\mu_{u}^{I^{\prime}}=-1 / 15, \mu_{v}^{I^{\prime}}=-15$, and $\varepsilon_{w}^{I^{\prime}}=-5 / 3$ for Segment I and $\mu_{u}^{I I^{\prime}}=-3, \mu_{v}^{I I^{\prime}}=-1 / 3$, and $\varepsilon_{w}^{I^{\prime}}=-3$ for Segment II. And the inner square core is filled with a homogeneous material with $\varepsilon_{w}^{\text {core' }}=\mu_{w}^{\text {core' }}=$ 25. The current distribution of the small current plane in these two cases is assumed $J_{s}=5 \exp \left(-400 y^{2}\right)$. Moreover, the corresponding field distribution of a large-sized current plane with the width $0.8 \mathrm{~m}$ radiating in free space is shown in Figure 2(c). Note that the current distribution would change the form under the transformation characterized by $J_{s}^{\prime}=$ $\exp \left(-16 y^{2}\right)$. The simulated results demonstrate that the small current plane coating with the polygonal metamaterial shell has the identical radiation characteristic as that of a large one. However, the antenna aperture is suppressed; that is, the size of the antenna aperture in Figure 2(b) is $0.354 \mathrm{~m}$, while the size of the antenna aperture in Figure $2(\mathrm{c})$ is $0.4 \mathrm{~m}$. Note that if a higher compressing ratio, that is, $r_{2} / r_{1}$, is used in the transformation optics, the antenna size can be further suppressed.

This concept can also be applied to reduce the size of antenna arrays that are usually adopted in highly directional radiating scheme. As a theoretical verification, we consider a nine-element antenna array of which the current distribution is set to be $1: 8: 28: 56: 70: 56: 28: 8: 1$ to achieve high directivity. The electric field distribution of the small-size antenna array coating with metamaterial shell is shown in Figure 3(a). The distance between two neighboring elements is $1.875 \mathrm{~cm}$, one-eighth of the wavelength. For comparison, Figure 3(b) illustrates the associated field distribution for a large-size one with a neighboring distance of $9.375 \mathrm{~cm}$. The radiation characteristic remains unchanged when replacing the large antenna array with a small-size one coated with properly designed metamaterial substrates.

It should be noted that this concept can be extended to other shapes of the antenna design. To further show the flexibility of such linear homogenous transformation approach in antenna design, we employ a hexagonal counterpart for comparison. As shown in Figure 4(a), the hexagonal metamaterial shell comprises of six yellow triangles and six blue triangles, marked as Segment I and Segment II, respectively. Different from the square case, the parameters we adopt here are $r_{0}=\sqrt{2} / 10 \mathrm{~m}, r_{1}=2 r_{0}$, and $r_{2}=4 r_{0}$. The electric field distribution of a small antenna array embedded in the hexagonal metamaterial shell is displayed in Figure 4(b). Here, the distance between the neighboring antenna elements is $1.875 \mathrm{~cm}$, identical to the square case. Similarly, Figure 4(c) depicts the radiation characteristic of the mentioned current plane embedded in such hexagonal metamaterial shell. The field distributions of equivalent large size antenna array 


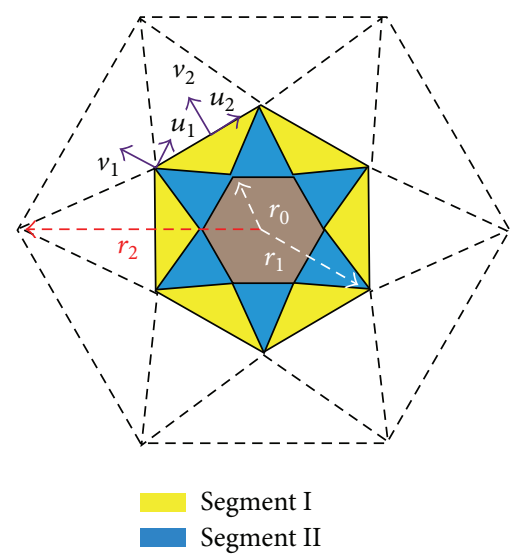

(a)

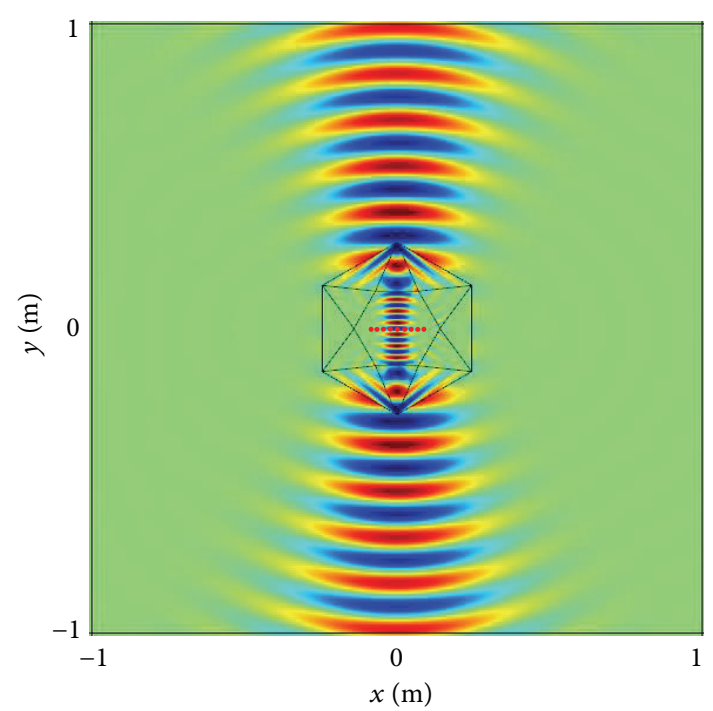

(b)

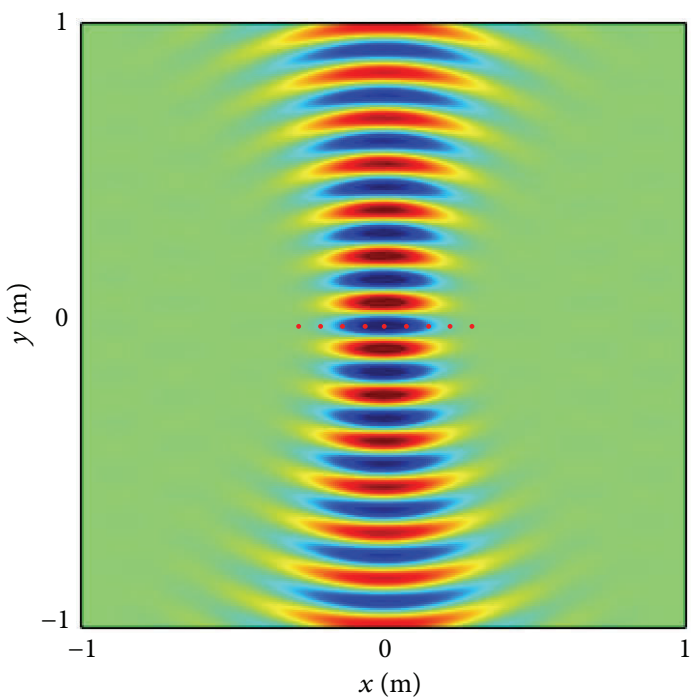

(d)

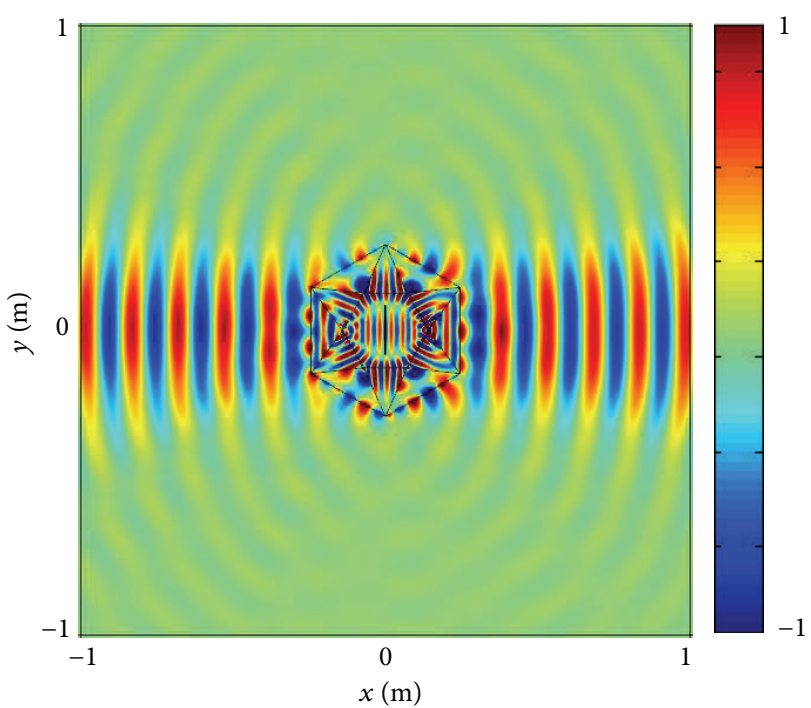

(c)

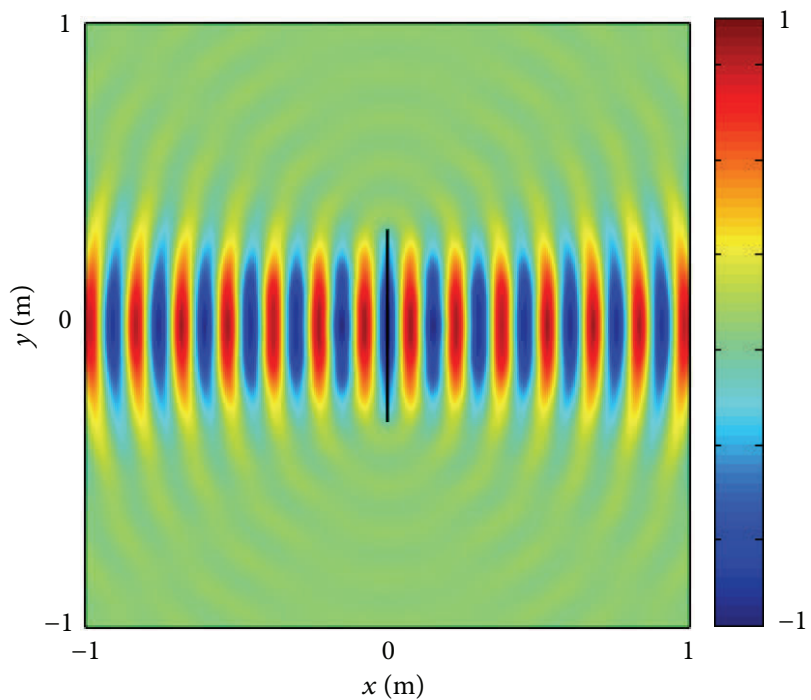

(e)

FIGURE 4: (a) The structure of a hexagonal metamaterial shell. (b) $E_{z}$ field distribution of a small antenna array with nine elements embedded in hexagonal metamaterial shell. (c) $E_{z}$ field distribution of a small current plane embedded in hexagonal metamaterial shell. The $z$-directed electrical field distributions of (d) large size antenna array and (e) large size current plane in free space. 
and current plane are displayed in Figures 4(d) and 4(e), respectively. These simulated results effectively prove our methodology an ideal candidate for the miniaturization of antenna system.

It should be noted that, in previous work based on inhomogeneous coordinate transformation [13], the values of the consitiutive paramters of the metamaterial shell are described by a function dependent on the cylindrical radius and vary as the change of the coordinates. To achieve these stringent demands, the metamaterial substrates must be designed with gradually varied parameters and hence harsh technique requirments are needed in practice. Besides, the impedanc mismatch between neighbouring layers is inevitable, while using discretization approach and the total performance will be deteriorated. These features, therefore, are main bottlenecks in practical microwave applications.

Compared with inhomogeneous transform optics method, the antenna coating obtained from linear homogeneous coordinate transformation is composed of homogeneous metamaterials whose constitutive parameters are kept consistent in each triangle region. Thus the difficulty in implementation will be greatly reduced. Besides, the linear homogeneous coordinate transformation is valid for arbitrary regular polygon and hence provides much flexibility in shape design. Furthermore, the antenna coating comprised of several separated components will be convenient to transport and assemble outdoors and, therefore, greatly facilitates the practical applications.

\section{Conclusion}

In conclusion, we have analytically presented an effective way of using a linear homogeneous coordinate transformation to achieve highly directional emission with a small antenna aperture. Since previous inhomogeneous transformation method resorts to inhomogeneous materials requiring a high precise fabrication technique to implement, we divide the virtual space into triangle segments and transformed them separately to avoid the requirement of inhomogeneous metamaterials. This can greatly reduce the complexity of the antenna materials' parameters. Simulated results have shown that small antenna devices embedded in properly designed metamaterial shell demonstrate identical radiation characteristics compared with large-sized ones, showing the potential applications in microwave engineering.

\section{Conflict of Interests}

The authors declare that there is no conflict of interests regarding the publication of this paper.

\section{Acknowledgments}

This work was sponsored by the National Natural Science Foundation of China under Grants no. 61322501, no. 61275183, no. 60990320, and no. 60990322; the National Program for Special Support of Top-Notch Young Professionals; the Foundation for the Author of National Excellent
Doctoral Dissertation of PR China under Grant no. 200950; the Program for New Century Excellent Talents (NCET12-0489) in University; the K. P. Chao's High Technology Development Foundation; and the Fundamental Research Funds for the Central Universities (2014XZZX003-24).

\section{References}

[1] J. B. Pendry, "Negative refraction makes a perfect lens," Physical Review Letters, vol. 85, no. 18, pp. 3966-3969, 2000.

[2] N. Fang, H. Lee, C. Sun, and X. Zhang, "Sub-diffraction-limited optical imaging with a silver superlens," Science, vol. 308, no. 5721, pp. 534-537, 2005.

[3] J. B. Pendry, D. Schurig, and D. R. Smith, "Controlling electromagnetic fields," Science, vol. 312, no. 5781, pp. 1780-1782, 2006.

[4] U. Leonhardt, “Optical conformal mapping," Science, vol. 312, no. 5781, pp. 1777-1780, 2006.

[5] D. Schurig, J. J. Mock, B. J. Justice et al., "Metamaterial electromagnetic cloak at microwave frequencies," Science, vol. 314, no. 5801, pp. 977-980, 2006.

[6] Y. Lai, J. Ng, H. Chen et al., "Illusion optics: the optical transformation of an object into another object," Physical Review Letters, vol. 102, no. 25, Article ID 253902, 2009.

[7] J. Pendry, "Optics: all smoke and metamaterials," Nature, vol. 460, no. 7255, pp. 579-580, 2009.

[8] J. Ng, H. Y. Chen, and C. T. Chan, "Metamaterial frequencyselective superabsorber," Optics Letters, vol. 34, pp. 644-646, 2009.

[9] Y. Luo, J. J. Zhang, L. X. Ran, H. S. Chen, and J. A. Kong, "New concept conformal antennas utilizing metamaterial and transformation optics," IEEE Antennas and Wireless Propagation Letters, vol. 7, pp. 508-512, 2008.

[10] N. Kundtz, D. A. Roberts, J. Allen, S. Cummer, and D. R. Smith, "Optical source transformations," Optics Express, vol. 16, no. 26, pp. 21215-21222, 2008.

[11] J. Allen, N. Kundtz, D. A. Roberts, S. A. Cummer, and D. R. Smith, "Electromagnetic source transformations using superellipse equations," Applied Physics Letters, vol. 94, no. 19, Article ID 194101, 2009.

[12] B.-I. Popa, J. Allen, and S. A. Cummer, "Conformal array design with transformation electromagnetics," Applied Physics Letters, vol. 94, no. 24, Article ID 244102, 2009.

[13] Y. Luo, J. Zhang, H. Chen, J. Huangfu, and L. Ran, "Highdirectivity antenna with small antenna aperture," Applied Physics Letters, vol. 95, no. 19, Article ID 193506, 2009.

[14] H. Chen and B. Zheng, "Broadband polygonal invisibility cloak for visible light,” Scientific Reports, vol. 2, article 255, 2012. 

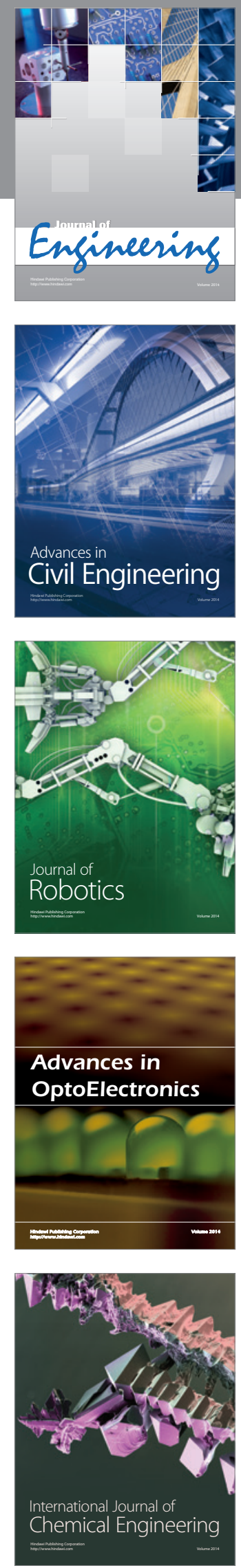

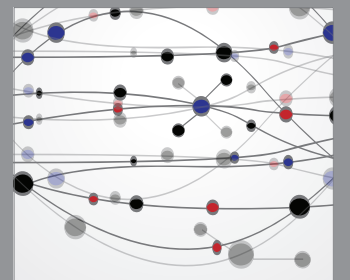

The Scientific World Journal
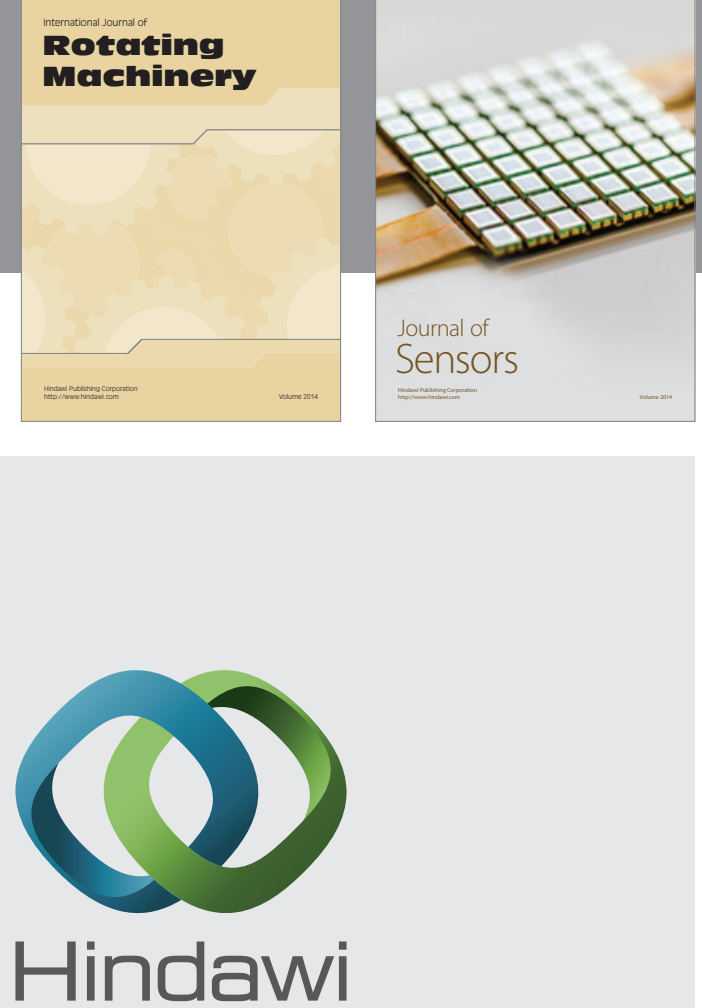

Submit your manuscripts at http://www.hindawi.com
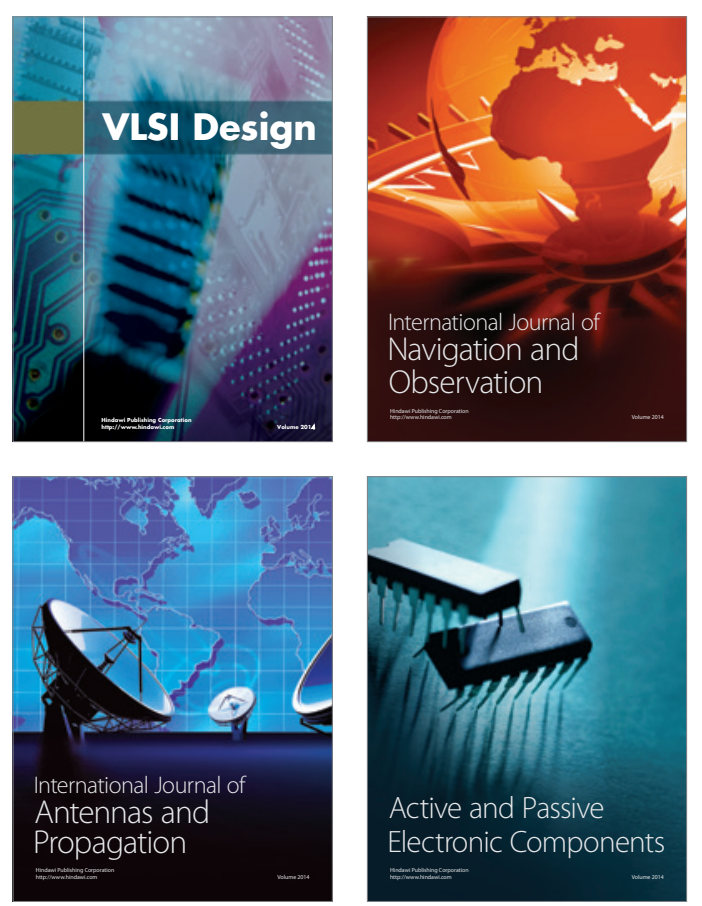
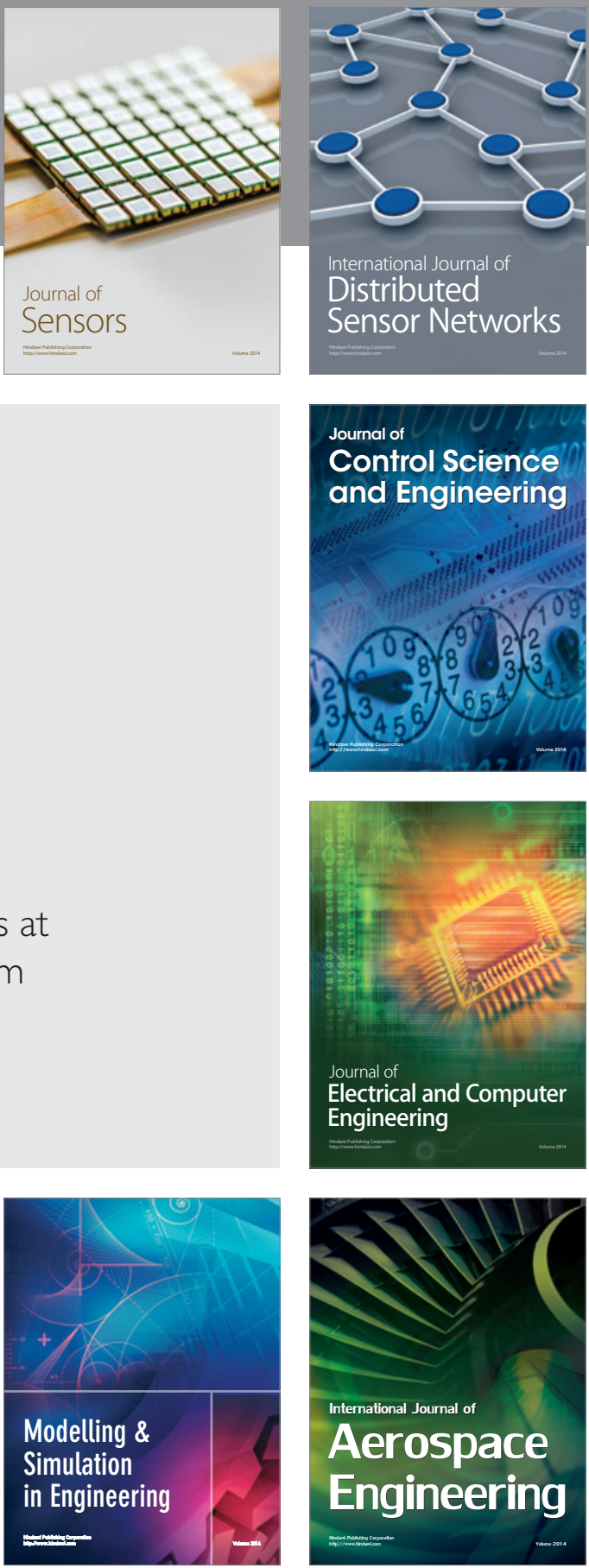

Journal of

Control Science

and Engineering
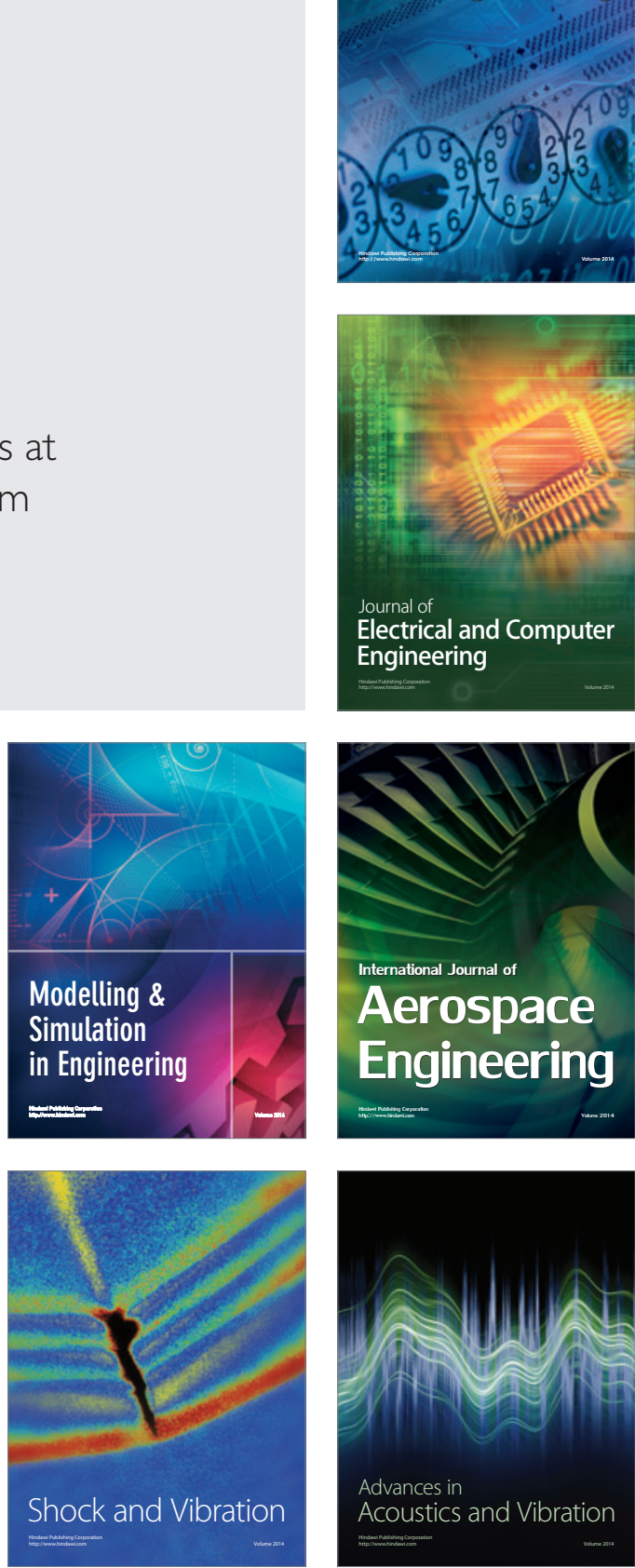